\title{
Magnification relations for Kerr lensing and testing Cosmic Censorship
}

\author{
M. C. Werner* \\ Institute of Astronomy, University of Cambridge, \\ Madingley Road, Cambridge CB3 OHA, United Kingdom \\ A. O. Petters ${ }^{\dagger}$ \\ Departments of Mathematics and Physics, Duke University, \\ Science Drive, Durham, NC 27708, United States of America
}

(Dated: 1 August 2007)

\begin{abstract}
A Kerr black hole with mass parameter $m$ and angular momentum parameter $a$ acting as a gravitational lens gives rise to two images in the weak field limit. We study the corresponding magnification relations, namely the signed and absolute magnification sums and the centroid up to post-Newtonian order. We show that there are post-Newtonian corrections to the total absolute magnification and centroid proportional to $a / m$, which is in contrast to the spherically symmetric case where such corrections vanish. Hence we also propose a new set of lensing observables for the two images involving these corrections, which should allow measuring $a / m$ with gravitational lensing. In fact, the resolution capabilities needed to observe this for the Galactic black hole should in principle be accessible to current and near-future instrumentation. Since $a / m>1$ indicates a naked singularity, a most interesting application would be a test of the Cosmic Censorship conjecture. The technique used to derive the image properties is based on the degeneracy of the Kerr lens and a suitably displaced Schwarzschild lens at post-Newtonian order. A simple physical explanation for this degeneracy is also given.

Version 2: to appear in Phys. Rev. D, http://prd.aps.org/, Copyright (2007) by the American Physical Society.
\end{abstract}

PACS numbers: 04.25.Nx, 04.70.Bw, 95.30.Sf, 98.62.Sb 


\section{INTRODUCTION}

The theory of gravitational lensing in the weak field limit has three physical inputs, namely perturbation theory of general relativity, geometric optics and the thin lens approximation $[1,2]$. Within this framework, a general formalism for lensing by spherically symmetric lenses up to post-post-Newtonian order in metric theories of gravity was recently developed by Keeton and Petters [3, 4]. Their appoach was extended to Kerr black holes by Sereno and de Luca [5] to study the effect of the angular momentum parameter on lensing properties.

We elaborate on this work in the present paper and determine the signed and total magnification sums of the images as well as the centroid for lensing by Kerr black holes in the weak field limit to post-Newtonian order. The post-Newtonian limit for rotating lenses was studied by Epstein and Shapiro [6] and in more detail by Sereno [7]. Correction terms for the Kerr black hole up to post-post-Newtonian order were derived by Sereno and de Luca [5], following earlier work by Bray [8], from the equations of motion for null geodesics in Kerr geometry. Since we are presently interested in the post-Newtonian limit only, a considerably simpler method to derive image positions and magnifications can be applied, based on the analysis by Asada, Kasai, and Yamamoto [9]. This utilizes the degeneracy of rotating lenses and displaced non-rotating lenses at this order, shown to hold generally by Asada and Kasai [10]. We provide a simple physical explanation for this degeneracy in the case of Kerr black holes in the weak field limit in Sec. II, then use the degeneracy explicitly to rederive image properties and find the new magnification relations in Sec. III. Based on this, we find new lensing observables for the two images involving post-Newtonian terms with $a / m$ in Sec. IV.

This introduces a lensing technique to measure angular momentum parameters of rotating black holes, which could complement spectroscopic and photometric studies to this end. For instance, the supermassive black hole at the Galactic center ( $\operatorname{Sgr} \mathrm{A}^{*}$ ) shows flares in X-ray, infrared and radio bands with polarization and quasi-periodic ( $\geq 13 \pm 2$ mins) substructure. Now this timescale appears to be associated with the innermost stable circular orbit, setting a lower limit of $a / m \geq 0.70 \pm 0.11$ (cf. [11] and the discussion therein). Measurements of

*Electronic address: mcw36@ast.cam.ac.uk

$\dagger$ Electronic address: petters@math.duke.edu 
$a / m$ are important, in particular, since they indicate whether Kerr black holes have naked singularities, which is the case if $a / m>1$. The absence of naked singularities in nature is stipulated by the Cosmic Censorship conjecture which is evoked in the singularity theorems (see Penrose [12] and references therein).

Hence the main application we have in mind is a lensing test of the Cosmic Censorship conjecture. Lensing properties of spherically symmetric static naked singularities were investigated by Virbhadra and Ellis [13]. More recently, Keeton and Petters [3] explored how lensing by the spherically symmetric Reissner-Nordstrøm and Gibbons-Maeda-GarfinkleHorowitz-Strominger black holes can be used to test the Cosmic Censorship conjecture. Here, however, we extend this work to the non-spherically symmetric but astrophysically more realistic Kerr black holes.

With regard to conventions, the metric signature $(-,+,+,+)$ is employed, and full units are used, where $G$ is the gravitational constant and $c$ is speed of light, to facilitate observational applications. The mass parameter $m=G M_{\bullet} / c^{2}$ is the gravitational radius, where $M_{\bullet}$ is the physical mass of the black hole, and the angular momentum parameter $a=J /(M \bullet c)$ is the specific angular momentum. Greek indices denote spacetime coordinates and Latin indices spatial coordinates.

\section{LENSING FRAMEWORK}

\section{A. Post-Newtonian formalism}

We begin by reviewing the post-Newtonian formalism for a Schwarzschild lens with mass parameter $m$ in view of the later application to the Kerr black hole. Let $\Theta=\left(\Theta_{1}, \Theta_{2}\right),|\Theta|=$ $\Theta$ and $\mathcal{B}=\left(\mathcal{B}_{1}, \mathcal{B}_{2}\right),|\mathcal{B}|=\mathcal{B}$ be the Cartesian angular coordinates in the lens plane and source plane, respectively, whose coordinate axes are parallel and whose origins are on the optical axis. The deflection angle projected into the lens plane is denoted by $\hat{\boldsymbol{\alpha}}=$ $\left(\hat{\alpha_{1}}, \hat{\alpha_{2}}\right),|\hat{\boldsymbol{\alpha}}|=\hat{\alpha}$. Then straightforward plane geometry in the standard lensing framework yields the lens equation [14]

$$
\tan \mathcal{B}=\tan \Theta-\frac{d_{L S}}{d_{S}}(\tan \Theta+\tan (\hat{\alpha}-\Theta)),
$$

where $d_{L}, d_{S}, d_{L S}$ denotes the angular diameter distances from the observer to lens and source plane, and from the lens to the source plane, respectively. Up to the post-Newtonian limit, 
the angular coordinates can be expressed in terms of dimensionless coordinates $\left(\mathcal{B}_{1}, \mathcal{B}_{2}\right)=$ $\theta_{E}\left(\beta_{1}, \beta_{2}\right),\left(\Theta_{1}, \Theta_{2}\right)=\theta_{E}\left(\theta_{1}, \theta_{2}\right)$ such that

$$
\begin{aligned}
& \mathcal{B}=\theta_{E} \beta=\theta_{E}\left(\beta_{(0)}+\beta_{(1)} \epsilon+\mathcal{O}\left(\epsilon^{2}\right)\right) \\
& \Theta=\theta_{E} \theta=\theta_{E}\left(\theta_{(0)}+\theta_{(1)} \epsilon+\mathcal{O}\left(\epsilon^{2}\right)\right),
\end{aligned}
$$

where the angular radius of the Einstein ring and the expansion parameter are

$$
\theta_{E}^{2}=\frac{4 m d_{L S}}{d_{L} d_{S}}, \quad \epsilon=\frac{\theta_{E} d_{S}}{4 d_{L S}}
$$

The impact paramter in the lens plane is $b=d_{L} \sin \Theta$ and the Schwarzschild deflection angle is

$$
\hat{\alpha}=4 \frac{m}{b}+\frac{15 \pi}{4} \frac{m^{2}}{b^{2}}+\mathcal{O}\left(\frac{m^{3}}{b^{3}}\right) .
$$

Hence (1) can be recast thus

$$
\beta=\theta-\frac{1}{\theta}-\frac{15 \pi}{16} \frac{\epsilon}{\theta^{2}}
$$

to obtain the lens equation for the Schwarzschild black hole up to post-Newtonian order.

\section{B. Kerr lensing}

The line element of the Kerr metric $g_{\mu \nu}^{K}$ in the Boyer-Lindquist coordinates $\{t, r, \vartheta, \varphi\}$ denoted by $x_{B L}^{\mu}$ is [15]

$$
\begin{aligned}
d s^{2} & =g_{\mu \nu}^{K} d x_{B L}^{\mu} d x_{B L}^{\nu}=-\left(1-\frac{2 m r}{\rho^{2}}\right) c^{2} d t^{2}-\frac{4 a m r \sin ^{2} \vartheta}{\rho^{2}} c d t d \varphi \\
& +\frac{\rho^{2}}{\Delta} d r^{2}+\rho^{2} d \vartheta^{2}+\frac{\left(r^{2}+a^{2}\right)^{2}-\Delta a^{2} \sin ^{2} \vartheta}{\rho^{2}} \sin ^{2} \vartheta d \varphi^{2}
\end{aligned}
$$

where $\rho^{2}=r^{2}+a^{2} \cos ^{2} \vartheta$ and $\Delta=r^{2}-2 m r+a^{2}$, which reduces to the Schwarzschild case if $a=0$. As mentioned above, the condition for a naked singularity is $a / m>1$ because then $\Delta>0$ and so no hypersurface $r=$ const. can be null which in turn means that no event horizon exists. For $a \neq 0$, the degeneracy of the central caustic point of the Schwarzschild lens is lifted to give rise to a central caustic domain bounded by a distorted astroid [16]. We are interested in the weak deflection limit and hence in the outer caustic domain where two images occur as in the Schwarzschild solution, albeit with modified positions and magnifications. In the standard gravitational lensing scenario, the null geodesics cross the equatorial 
plane $\vartheta=\pi / 2$ at least once. In terms of constants of motion in Kerr geometry, we therefore restrict this discussion to null geodesics with Carter constant $Q \geq 0$ [17, p. 205].

The lens plane coordinates introduced in the previous section can now be conveniently oriented so that the $\Theta_{2}$-axis is along the projected angular momentum axis and forms a right-handed system together with $\Theta_{1}$-axis and the optical axis, with the observer at $d_{L}$, as the third one. Now up to post-Newtonian order, Kerr lensing is equivalent to lensing by a Schwarzschild lens of the same mass but shifted to the position $[5,9,10,16]$

$$
\delta \boldsymbol{\Theta}=\theta_{E}\left(\delta \theta_{1}, 0\right)=\theta_{E}\left(\delta \theta_{1(1)} \epsilon, 0\right), \delta \theta_{1(1)}=\frac{a \sin \vartheta_{O}}{m},
$$

where $\vartheta_{O}$ is the observer's polar angle position.

We now show how this fact can be understood in a simple way in terms of the gravitomagnetic effect, and use it explicitly in the next section to find the corrected image positions and magnifications. For an extended discussion of the gravitomagnetic effect for the Kerr and more general rotating lenses, see Asada et al. [9, 10], Kopeikin et al. [18] and Sereno [19]. In the weak field limit, the metric $g_{\mu \nu}$ can be understood as a formal perturbation $h_{\mu \nu}$ about the Minkowski metric $\eta_{\mu \nu}$ such that $g_{\mu \nu}=\eta_{\mu \nu}+h_{\mu \nu},\left|h_{\mu \nu}\right|<<1$. Defining the tracereversed perturbation $\bar{h}_{\mu \nu}=h_{\mu \nu}-g_{\mu \nu} h_{\alpha \beta} \eta^{\alpha \beta} / 2$, Einstein's field equation may be written $\square \bar{h}_{\mu \nu}=-16 \pi G T_{\mu \nu} / c^{4}$ in the de Donder gauge $\bar{h}_{, \nu}^{\mu \nu}=0$, where $T^{\mu \nu}$ is the energy momentum tensor. Now with a perfect, non-relativistic fluid, the components of its retarded solution give rise to a scalar field $U \equiv-\bar{h}_{00} c^{2} / 4$, which is the Newtonian gravitational potential, and a vector potential with components $V^{i} \equiv \bar{h}_{0 i} c^{2}$. Hence the perturbed metric line element is

$$
d s^{2}=g_{\mu \nu} d x^{\mu} d x^{\nu}=-\left(1+\frac{2 U}{c^{2}}\right) c^{2} d t^{2}+\frac{2}{c} \sum_{i} V^{i} d x^{i} d t+\left(1-\frac{2 U}{c^{2}}\right) \sum_{i}\left(d x^{i}\right)^{2}
$$

where we work with spatially isotropic coordinates $\mathbf{x}=\left(x_{1}, x_{2}, x_{3}\right),|\mathbf{x}|=x$. Here $x_{1}$ is aligned with $\Theta_{1}$ and $x_{3}=0$ corresponds to the equatorial plane $\vartheta=\pi / 2$. The gradient operator for this coordinate system is denoted by $\nabla$. Also, let $\mathbf{a}=a \hat{\mathbf{x}}_{\mathbf{3}}$ where $\hat{\mathbf{x}}_{\mathbf{3}}$ is the unit vector in the $x_{3}$ direction.

The equation of motion for null geodesics parametrized with $q$ and with unit ray 3-vector k can now be obtained from (6) using Fermat's principle, yielding a gravitoelectric and gravitomagnetic contribution (e.g., [10]),

$$
c^{2} \frac{d \mathbf{k}}{d q}=-2 \nabla_{\perp} U+\mathbf{k} \times(\nabla \times \mathbf{V}),
$$


where the operator $\nabla_{\perp}$ selects the component of the gradient perpendicular to the unit vector $\mathbf{k}$ such that, for any scalar field $\phi(\mathbf{x}), \nabla_{\perp} \phi \equiv \nabla \phi-(\nabla \phi \cdot \mathbf{k}) \mathbf{k}=\mathbf{k} \times(\nabla \phi \times \mathbf{k})$. The forefactor of the gravitoelectric term is the well-known general relativistic correction of Newtonian light deflection. In this limit, the Kerr metric (4) becomes

$$
d s^{2}=-\left(1-\frac{2 m}{x}\right) c^{2} d t^{2}-\frac{4 a m}{x^{3}} c d t\left(x_{1} d x_{2}-x_{2} d x_{1}\right)+\left(1+\frac{2 m}{x}\right) \sum_{i}\left(d x^{i}\right)^{2}
$$

whence one can read off $\mathbf{V}=-2 G M_{\bullet} \mathbf{a} \times \mathbf{x} / x^{3}$ by comparison with (6).

We can now see that lensing due to a Kerr black hole at $\mathbf{x}=\mathbf{0}$ to post-Newtonian order is equivalent to a Schwarzschild lens displaced according to (5), that is, at $\delta \mathbf{x}=d_{L} \delta \boldsymbol{\Theta}$. Since this Schwarzschild lens has zero vector potential and $U=-G M_{\bullet} /|\mathbf{x}-\delta \mathbf{x}|$, the right-hand side of (7) becomes, by Taylor expansion to post-Newtonian order,

$$
-2 \nabla_{\perp} U(\mathbf{x}-\delta \mathbf{x})=-2 \nabla_{\perp} U(\mathbf{x})+2 \nabla_{\perp}(\nabla U \cdot \delta \mathbf{x})
$$

because $\delta \mathbf{x}=\mathcal{O}(\epsilon)$ from (5), and the dot product is with respect to the Euclidean metric on the spatially isotropic coordinates. Furthermore, in the thin lens approximation, one may take $\mathbf{k}$ to be constant and perpendicular to the lens plane $L$ until $\mathbf{k}$ is changed by some $\delta \mathbf{k}$ upon crossing $L$. Since we consider the weak field limit, $|\delta \mathbf{k}|<<|\mathbf{k}|$ so the leading post-Newtonian term is

$$
2 \nabla_{\perp}(\nabla U \cdot \delta \mathbf{x})=2 \mathbf{k} \times(\nabla(\nabla U \cdot \delta \mathbf{x}) \times \mathbf{k})=\mathbf{k} \times(\nabla \times(2 \nabla U \cdot \delta \mathbf{x}) \mathbf{k}),
$$

which is indeed a gravitomagnetic term of the form occuring in (7). Hence the displaced Schwarzschild lens is equivalent to a point lens of mass $M_{\bullet}$ at $\mathbf{x}=\mathbf{0}$ with vector potential $2 G M_{\bullet} a \sin \vartheta_{O} x_{1} \mathbf{k} / x^{3}$, using (5) and (2). But $\mathbf{k}=\left(0,-\sin \vartheta_{O}, \cos \vartheta_{O}\right)$ by setup, so using the

expression for $\mathbf{V}$ above we find that this vector potential component is precisely provided by a Kerr black hole situated at the origin with angular momentum parameter $a$, as required.

\section{MAGNIFICATION RELATIONS}

\section{A. Image properties}

Following the discussion in the previous section, one can use the Schwarzschild lens equation to generate image properties of Kerr lensing up to post-Newtonian order in the weak 
field limit, where source and observer are in the asymptotically flat region with the source behind the lens plane and close to the optical axis. Given the shift (5), we need to let $\theta_{1} \mapsto \theta_{1}-\delta \theta_{1}$ in the last two terms of (3), which stem from the deflection angle of the lens model. Hence

$$
\begin{aligned}
& \beta_{1}=\theta_{1}-\frac{\theta_{1}-\delta \theta_{1}}{\left(\theta_{1}-\delta \theta_{1}\right)^{2}+\theta_{2}^{2}}-\frac{15 \pi}{16} \frac{\theta_{1}-\delta \theta_{1}}{\left(\left(\theta_{1}-\delta \theta_{1}\right)^{2}+\theta_{2}^{2}\right)^{3 / 2}} \epsilon+\mathcal{O}\left(\epsilon^{2}\right) \\
& \beta_{2}=\theta_{2}-\frac{\theta_{2}}{\left(\theta_{1}-\delta \theta_{1}\right)^{2}+\theta_{2}^{2}}-\frac{15 \pi}{16} \frac{\theta_{2}}{\left(\left(\theta_{1}-\delta \theta_{1}\right)^{2}+\theta_{2}^{2}\right)^{3 / 2}} \epsilon+\mathcal{O}\left(\epsilon^{2}\right)
\end{aligned}
$$

is our ansatz for the Kerr lens equation. Notice that, at Newtonian order, (9) reduces to the Schwarzschild lens equation (3) for $\epsilon=0$ as expected, since $\delta \theta_{1}=\mathcal{O}(\epsilon)$ according to (5). The expansion of the image positions is

$$
\begin{aligned}
& \theta_{1}=\theta_{1(0)}+\theta_{1(1)} \epsilon+\mathcal{O}\left(\epsilon^{2}\right) \\
& \theta_{2}=\theta_{2(0)}+\theta_{2(1)} \epsilon+\mathcal{O}\left(\epsilon^{2}\right)
\end{aligned}
$$

where $\theta_{1(0)}, \theta_{2(0)}$ solve the lens equation at Newtonian order. This yields the two images of the well-known Schwarzschild case, one of positive and one of negative parity,

$$
\begin{aligned}
& \theta_{1(0)}^{ \pm}=\frac{\beta_{1}}{2}\left(1 \pm \sqrt{1+\frac{4}{\beta^{2}}}\right) \\
& \theta_{2(0)}^{ \pm}=\frac{\beta_{2}}{2}\left(1 \pm \sqrt{1+\frac{4}{\beta^{2}}}\right)
\end{aligned}
$$

where $\theta_{1(0)}^{2}+\theta_{2(0)}^{2}=\theta_{(0)}^{2}$. Now at post-Newtonian order, the lens equation (9) becomes

$$
\begin{aligned}
& 0=\theta_{1(1)}+\theta_{1(0)}\left(\frac{A}{\theta_{(0)}^{4}}-\frac{15 \pi}{16 \theta_{(0)}^{3}}\right)-\frac{\theta_{1(1)}-\delta \theta_{1(1)}}{\theta_{(0)}^{2}} \\
& 0=\theta_{2(1)}+\theta_{2(0)}\left(\frac{A}{\theta_{(0)}^{4}}-\frac{15 \pi}{16 \theta_{(0)}^{3}}\right)-\frac{\theta_{2(1)}}{\theta_{(0)}^{2}}
\end{aligned}
$$

where $A=2\left(\theta_{1(1)}-\delta \theta_{1(1)}\right) \theta_{1(0)}+2 \theta_{2(1)} \theta_{2(0)}$, and we recover the correction terms expected for rotating lenses [5],

$$
\begin{aligned}
& \theta_{1(1)}=\frac{15 \pi \theta_{1(0)}}{16\left(1+\theta_{(0)}^{2}\right) \theta_{(0)}}+\frac{\left(1-\theta_{1(0)}^{2}+\theta_{2(0)}^{2}\right) \delta \theta_{1(1)}}{1-\theta_{(0)}^{4}} \\
& \theta_{2(1)}=\frac{15 \pi \theta_{2(0)}}{16\left(1+\theta_{(0)}^{2}\right) \theta_{(0)}}-\frac{2 \theta_{1(0)} \theta_{2(0)} \delta \theta_{1(1)}}{1-\theta_{(0)}^{4}} .
\end{aligned}
$$

Accordingly, the individual post-Newtonian corrections for the positive and the negative parity image are found by substituting $\theta_{1(0)}^{ \pm}, \theta_{2(0)}^{ \pm}$from (11) into (12). For a discussion and visualization of this shift, see Sereno [7], especially his Figure 5. 
Since light rays are conserved in geometric optics, the signed image magnification $\mu$ is related to the Jacobian of the lens map [1, 2],

$$
\frac{1}{\mu}=\operatorname{det}\left(\begin{array}{cc}
\frac{\partial \beta_{1}}{\partial \theta_{1}} & \frac{\partial \beta_{1}}{\partial \theta_{2}} \\
\frac{\partial \beta_{2}}{\partial \theta_{1}} & \frac{\partial \beta_{2}}{\partial \theta_{2}}
\end{array}\right)
$$

Recall also that the observable image flux $F_{O}$ and the flux of the unlensed source $F_{S}$ are related by $F_{O}=|\mu| F_{S}$. Now evaluating the magnification yields

$$
\mu=\frac{\theta_{(0)}^{4}}{\theta_{(0)}^{4}-1}-\left(\frac{15 \pi \theta_{(0)}^{3}}{16\left(1+\theta_{(0)}^{2}\right)^{3}}-\frac{4 \theta_{(0)}^{4} \theta_{1(0)} \delta \theta_{1(1)}}{\left(1-\theta_{(0)}^{2}\right)^{2}\left(1+\theta_{(0)}^{2}\right)^{3}}\right) \epsilon+\mathcal{O}\left(\epsilon^{2}\right)
$$

which, up to a sign, coincides with the findings in [5]. Again, this expression holds for both images.

\section{B. Magnification sums}

The magnification formula (13) can now be used together with (11) and (12) to write down a new expression for the individual magnifications of the positive and negative parity image, respectively, to post-Newtonian order,

$$
\begin{aligned}
& \mu^{+}=\frac{\left(\beta+\sqrt{4+\beta^{2}}\right)^{4}}{\left(\beta+\sqrt{4+\beta^{2}}\right)^{4}-16}-\frac{\left(2+\beta^{2}+\beta \sqrt{4+\beta^{2}}\right)\left(15 \pi \beta^{3}-64 \beta_{1} \delta \theta_{1(1)}\right)}{8 \beta^{3}\left(\beta+\sqrt{4+\beta^{2}}\right)^{2}\left(4+\beta^{2}\right)^{3 / 2}} \epsilon+\mathcal{O}\left(\epsilon^{2}\right) \\
& \mu^{-}=\frac{\left(\beta-\sqrt{4+\beta^{2}}\right)^{4}}{\left(\beta-\sqrt{4+\beta^{2}}\right)^{4}-16}-\frac{\left(2+\beta^{2}-\beta \sqrt{4+\beta^{2}}\right)\left(15 \pi \beta^{3}+64 \beta_{1} \delta \theta_{1(1)}\right)}{8 \beta^{3}\left(\beta-\sqrt{4+\beta^{2}}\right)^{2}\left(4+\beta^{2}\right)^{3 / 2}} \epsilon+\mathcal{O}\left(\epsilon^{2}\right) .
\end{aligned}
$$

Hence the sum of the signed magnifications can be evaluated and is of the simple form

$$
\mu^{+}+\mu^{-}=1-\frac{15 \pi}{8\left(4+\beta^{2}\right)^{3 / 2}} \epsilon+\mathcal{O}\left(\epsilon^{2}\right) .
$$

The Schwarzschild lens obeys a well-known magnification invariant (e.g., [2, p. 191]) in the standard lensing framework, that is, at lowest order. Since the signed magnification sum (15) for the Kerr lens does not depend on the specific angular momentum $a$, it is identical to the Schwarzschild lens result to post-Newtonian order (cf. Eq. (54) of [4]). The Kerr lens has thus the same deviation from the magnification invariant as the Schwarzschild lens at $\mathcal{O}(\epsilon)$.

Now taking into account the image parities, the absolute magnifications are $\left|\mu^{+}\right|=\mu^{+}$ and $\left|\mu^{-}\right|=-\mu^{-}$. Hence, the total absolute magnification is

$$
\mu_{\mathrm{tot}}=\left|\mu^{+}\right|+\left|\mu^{-}\right|=\frac{2+\beta^{2}}{\beta \sqrt{4+\beta^{2}}}+\frac{8 \beta_{1}}{\beta^{3}\left(4+\beta^{2}\right)^{3 / 2}} \frac{a \sin \vartheta_{O}}{m} \epsilon+\mathcal{O}\left(\epsilon^{2}\right)
$$


using (2). The term $\mathcal{O}(\epsilon)$ vanishes for $a=0$ or an observer on the rotational axis of the Kerr black hole, that is $\vartheta_{O}=0$, as expected for circularly symmetric lenses [3].

\section{Centroid}

We can also define the centroid of the magnification thus,

$$
\Theta^{\text {Cent }}=\theta_{E} \frac{\boldsymbol{\theta}^{+}\left|\mu^{+}\right|+\boldsymbol{\theta}^{-}\left|\mu^{-}\right|}{\left|\mu^{+}\right|+\left|\mu^{-}\right|} .
$$

Given (11), (12) and (14), a new expression for the centroid vector of Kerr images to postNewtonian order can now be obtained, and its components turn out to be

$$
\begin{aligned}
& \Theta_{1}^{\text {Cent }}=\theta_{E}\left[\frac{\left(3+\beta^{2}\right) \beta_{1}}{2+\beta^{2}}+\frac{\left(\beta_{1}^{2}-\beta_{2}^{2}-2\right)}{\left(2+\beta^{2}\right)^{2}} \frac{a \sin \vartheta_{O}}{m} \epsilon+\mathcal{O}\left(\epsilon^{2}\right)\right] \\
& \Theta_{2}^{\text {Cent }}=\theta_{E}\left[\frac{\left(3+\beta^{2}\right) \beta_{2}}{2+\beta^{2}}+\frac{2 \beta_{1} \beta_{2}}{\left(2+\beta^{2}\right)^{2}} \frac{a \sin \vartheta_{O}}{m} \epsilon+\mathcal{O}\left(\epsilon^{2}\right)\right] .
\end{aligned}
$$

Again, in the circularly symmetric case $a=0$ or $\vartheta_{O}=0$ we can take $\beta_{2}=0$ without loss of generality, to recover the result by Keeton and Petters [3].

\section{APPLICATIONS}

\section{A. Breaking the degeneracy}

Asada and Kasai [9] found that, at post-Newtonian order, rotating and non-rotating dark lenses cannot be distinguished on account of the degeneracy discussed in Sec. II, that is, by observing the images alone. This problem can be circumvented if the location of the black hole is established independently, for instance by observing the center of the accretion disk surrounding the Kerr black hole. To see this, recall from Sec. II that the Kerr lens $K$ is equivalent to a displaced Schwarzschild lens up to post-Newtonian order in the weak field limit where observer and source are assumed to be in the asymptotically flat region of the Kerr black hole. Hence a plane $P$ containing the observer, the source and the notional shifted Schwarzschild lens will also contain the two images of the Kerr lens as for a standard Schwarzschild lens. Projected into the plane of the sky, the source, the notional shifted Schwarzschild lens and the two images will be collinear but not typically with $K$ since $K \notin P$ in general. Hence, if the position of $K$ can be found independently, the projected 
distance of $K$ from the line joining the two images is observable and the degeneracy is broken in the generic case. However, note for completeness that there are very special cases in which the degeneracy cannot be broken in this way: consider a source such that $\mathcal{B}_{2}=0$ exactly, so $\theta_{2(0)}=0$ and hence $\theta_{2(1)}=0$ from (12). In this case, $K \in P$ and so the projected source, shifted Schwarzschild lens position, and the two images will all be collinear with $K$ at the origin. Therefore, the degeneracy is not broken.

But assuming the Kerr/Schwarzschild degeneracy is broken successfully, we still need to be able to measure image positions in the $\left(\Theta_{1}, \Theta_{2}\right)$ coordinate system in order to apply the formalism. Hence the direction of the Kerr black hole's spin axis projected into the lens plane must also be known and, moreover, the observer's $\vartheta_{O}$ coordinate. In principle, this could be inferred from observations of the jet associated with the black hole accretion disk because of the frame-dragging effect on the magnetohydrodynamics of the jet (e.g., [20]). Furthermore, time-dependent measurements of the polarization of black hole flare emission could constrain the direction of the spin axis. In the case of $\operatorname{Sgr} \mathrm{A}^{*}$, for instance, this seems to indicate that the black hole spin axis is essentially aligned with the Galaxy's [11].

\section{B. Measuring the angular momentum parameter}

In order to determine whether Kerr lensing could be used to measure $a / m$ and test the Cosmic Censorship conjecture, we first of all need to assemble a suitable set of observables. This set is in turn dependent on resolution capability: If the two Kerr images can in fact be resolved, then image positions and fluxes will be observable individually. If, however, they cannot be resolved, the total flux and the magnification centroid may be taken as observables. We shall discuss the former case first.

If the two images can be resolved, two vectorial image positions and two fluxes are available, giving six equations altogether. Using (10-12) and (15-16), we propose the following combinations as convenient observables,

$$
\begin{aligned}
\Theta_{1}^{+}+\Theta_{1}^{-} & =\frac{\mathcal{B}_{1}}{\sqrt{4 \theta_{E}^{2}+\mathcal{B}^{2}}}\left(\sqrt{4 \theta_{E}^{2}+\mathcal{B}^{2}}-\frac{15 \pi \theta_{E}}{16} \epsilon\right)+\frac{a \sin \vartheta_{O} \theta_{E}}{m} \epsilon+\mathcal{O}\left(\epsilon^{2}\right) \\
\Theta_{2}^{+}+\Theta_{2}^{-} & =\frac{\mathcal{B}_{2}}{\sqrt{4 \theta_{E}^{2}+\mathcal{B}^{2}}}\left(\sqrt{4 \theta_{E}^{2}+\mathcal{B}^{2}}-\frac{15 \pi \theta_{E}}{16} \epsilon\right)+\mathcal{O}\left(\epsilon^{2}\right) \\
\Theta_{1}^{+}-\Theta_{1}^{-} & =\frac{\mathcal{B}_{1}}{\mathcal{B}}\left(\sqrt{4 \theta_{E}^{2}+\mathcal{B}^{2}}+\frac{15 \pi \theta_{E}}{16} \epsilon\right)-\frac{\mathcal{B}^{4} \theta_{E}+4 \mathcal{B}_{2}^{2} \theta_{E}^{3}}{\mathcal{B}^{3} \sqrt{4 \theta_{E}^{2}+\mathcal{B}^{2}}} \frac{a \sin \vartheta_{O}}{m} \epsilon+\mathcal{O}\left(\epsilon^{2}\right)
\end{aligned}
$$




$$
\begin{aligned}
& \Theta_{2}^{+}-\Theta_{2}^{-}=\frac{\mathcal{B}_{2}}{\mathcal{B}}\left(\sqrt{4 \theta_{E}^{2}+\mathcal{B}^{2}}+\frac{15 \pi \theta_{E}}{16} \epsilon\right)+\frac{4 \mathcal{B}_{1} \mathcal{B}_{2} \theta_{E}^{3}}{\mathcal{B}^{3} \sqrt{4 \theta_{E}^{2}+\mathcal{B}^{2}}} \frac{a \sin \vartheta_{O}}{m} \epsilon+\mathcal{O}\left(\epsilon^{2}\right) \\
& F_{O}^{+}+F_{O}^{-}=F_{S}\left(\frac{2 \theta_{E}^{2}+\mathcal{B}^{2}}{\mathcal{B} \sqrt{4 \theta_{E}^{2}+\mathcal{B}^{2}}}+\frac{8 \mathcal{B}_{1} \theta_{E}^{5}}{\mathcal{B}^{3}\left(4 \theta_{E}^{2}+\mathcal{B}^{2}\right)^{3 / 2}} \frac{a \sin \vartheta_{O}}{m} \epsilon\right)+\mathcal{O}\left(\epsilon^{2}\right) \\
& F_{O}^{+}-F_{O}^{-}=F_{S}\left(1-\frac{15 \pi \theta_{E}^{3}}{8\left(4 \theta_{E}^{2}+\mathcal{B}^{2}\right)^{3 / 2}} \epsilon\right)+\mathcal{O}\left(\epsilon^{2}\right)
\end{aligned}
$$

which reduce to the formulae of Keeton and Petters [4] for $a=0$ or $\sin \vartheta_{O}=0$, as required. These six equations could then be solved for the six occuring variables $\mathcal{B}_{1}, \mathcal{B}_{2}, F_{S}, \theta_{E}, \epsilon, a \sin \vartheta_{O} / m$. Assuming that the lensed source orbits the black hole such that $d_{L S}<<d_{L}$ and that an independent estimate for $m$ is available, then $d_{L S}$ and $d_{L} \approx d_{S}$ can also be found from $\theta_{E}$ and $\epsilon$ using (2).

It would therefore be possible to infer $a / m$ from the post-Newtonian lensing corrections. Moreover, these corrections should in principle be observable with near-future instrumentation as discussed by Keeton and Petters [4]. In the case of Sgr A*, they found an estimate for the angular Einstein radius to be of order $\theta_{E}=0.022\left(d_{L S} / 1 \mathrm{pc}\right)^{1 / 2}$ arcsec and perturbation parameter to be of order $\epsilon=2.1 \times 10^{-4} \times\left(d_{L S} / 1 \mathrm{pc}\right)^{1 / 2}$. Since the image separations will be of order $\theta_{E}$, these images can in principle be resolved with current technology (e.g., the CHARA interferometer and radio interferometry can resolve $10^{-3}$ arcsec separations [21]). Furthermore, currently known positional uncertainties in observed radio images are of order $10^{-6}$ arcsec (e.g., [22]). These are indeed much smaller than the current resolution capabilities. In addition, the statistical prospects for observing lensed stars around Sgr A* are discussed in a forthcoming paper by Congdon et al. [23] who conclude that the disk component of the Milky Way contributes more than the bulge, and find that the expected number of lenses reaches unity for a detection limit of $K \sim 18.5$ mag.

In the case when the two images cannot be resolved, then only the total flux (22) and the centroid (17) are available. In the forseeable future, this situation applies to lensing by extragalactic supermassive black holes, and Congdon et al. [23] estimate that typically $\sim 100$ lensed stars can be expected. But given that we only have three equations for six variables in this case, a determination of $a / m$ does not seem possible. However, this situation may improve if additional information, for instance on $m, d_{L}$ and $F_{S}$, becomes available.

Finally, we should stress again that the success of this method is conditional, in both cases, upon breaking the Kerr/Schwarzschild degeneracy and establishing the $\Theta_{1}, \Theta_{2}, \vartheta_{O}$ coordinates, as discussed in the previous section. Further data, for example the time delay 
between images of a variable source (cf. [4]) or images of multiple sources, may also be helpful for breaking degeneracies. Since equations (18 - 23) fully determine the six occurring variables, we have not considered these ramifications here. Nonetheless, our analysis shows that lensing measurements of $a / m$ for supermassive black holes, and hence lensing tests of Cosmic Censorship, have potential.

\section{CONCLUSION}

We considered gravitational lensing in the weak field limit of a Kerr black hole of mass parameter $m$ and angular momentum parameter $a$ and derived the magnification relations for the two ensuing images up to post-Newtonian order. The image properties used were rederived with a simple perturbation analysis based on the degeneracy of a Kerr lens and a Schwarzschild lens shifted by (5). Whereas the signed magnification sum (15) turned out to be identical to the Schwarzschild case, the absolute magnification sum (16) and centroid (17) show a term proportional to $a / m$ at post-Newtonian order. This is in contrast to circularly symmetric lenses where these terms have been shown to vanish precisely. In discussing observational implications, we provided a new set of six lensing observables (18 - 23) for the case that the two images can be resolved. These are matched with six lensing variables including $a \sin \vartheta_{O} / m$. In the case of lensing by the Galactic black hole, the two images should be resolvable by current and near-future instrumentation, so that measurements of the angular momentum parameter should be feasible. Since $a / m>1$ for naked singularities, this provides a possible test of the Cosmic Censorship conjecture using gravitational lensing. Additional study of this issue is definitely warranted.

\section{Acknowledgments}

MCW would like to thank the Department of Mathematics, Duke University, for their hospitality and gratefully acknowledges funding by the STFC (formerly PPARC), United Kingdom. AOP strongly acknowledges the research funding support of NSF Grants DMS- 
0302812, AST-0434277, and AST-0433809.

[1] P. Schneider, J. Ehlers and E. E. Falco, Gravitational lenses (Springer, Berlin, 1992)

[2] A. O. Petters, H. Levine, and J. Wambsganss, Singularity theory and gravitational lensing (Birkhäuser, Boston, 2001)

[3] C. R. Keeton and A. O. Petters, Phys. Rev. D 72, 104006 (2005)

[4] C. R. Keeton and A. O. Petters, Phys. Rev. D 73, 044024 (2006)

[5] M. Sereno and F. De Luca, Phys. Rev. D 74, 123009 (2006)

[6] R. Epstein and I. I. Shapiro, Phys. Rev. D 22, 2947 (1980)

[7] M. Sereno, Mon. Not. R. Astron. Soc. 344, 942 (2003)

[8] I. Bray, Phys. Rev. D 34, 367 (1986)

[9] H. Asada, M. Kasai and T. Yamamoto, Phys. Rev. D 67, 043006 (2003)

[10] H. Asada and M. Kasai, Prog. Theor. Phys. 104, 95 (2000)

[11] S. Trippe, et al., Mon. Not. R. Astron. Soc. 375, 764 (2007)

[12] R. Penrose, J. Astrophys. Astr. 20, 233 (1999)

[13] K. S. Virbhadra and G. F. R. Ellis, Phys. Rev. D 65, 103004 (2002)

[14] K. S. Virbhadra and G. F. R. Ellis, Phys. Rev. D 62, 084003 (2000)

[15] S. Chandrasekhar, The mathematical theory of black holes (Clarendon, Oxford, 1983)

[16] K. P. Rauch and R. D. Blandford, Astrophys. J. 421, 46 (1994)

[17] B. O'Neill, The geometry of Kerr black holes (Peters, Wellesley MA, 1995)

[18] S. Kopeikin and B. Mashhoon, Phys. Rev. D 65, 064025 (2002)

[19] M. Sereno, Phys. Lett. A 305, 7 (2002)

[20] R. Narayan and E. Quataert, Science 307, 77 (2005)

[21] T. A. ten Brummelaar, et al., Astrophys. J. 628, 453 (2005)

[22] C. S. Trotter, J. N. Winn and J. N. Hewitt, Astrophys. J. 535, 671 (2000)

[23] A. B. Congdon, C. R. Keeton and C. E. Nordgren, Phys. Rev. D, submitted 